\title{
Self-organized bistability and its possible relevance for brain dynamics
}

\author{
Victor Buendía $\odot,{ }^{1,2,3}$ Serena di Santo, ${ }^{4}$ Pablo Villegas $\odot,{ }^{5}$ Raffaella Burioni, ${ }^{2,3}$ and Miguel A. Muñoz $\circledast^{1,2}$ \\ ${ }^{1}$ Departamento de Electromagnetismo y Física de la Materia e Instituto Carlos I de Física Teórica y Computacional, \\ Universidad de Granada, E-18071 Granada, Spain \\ ${ }^{2}$ Dipartimento di Scienze Matematiche, Fisiche e Informatiche, Università di Parma, via G.P. Usberti, 7/A - 43124, Parma, Italy \\ ${ }^{3}$ INFN, Gruppo Collegato di Parma, via G.P. Usberti, 7/A - 43124, Parma, Italy \\ ${ }^{4}$ Morton B. Zuckerman Mind Brain Behavior Institute Columbia University, 10027 New York, USA \\ ${ }^{5}$ Istituto dei Sistemi Complessi, CNR, via dei Taurini 19, 00185 Rome, Italy
}

(Received 13 November 2019; accepted 21 February 2020; published 16 March 2020)

\begin{abstract}
Self-organized bistability (SOB) is the counterpart of "self-organized criticality" (SOC), for systems tuning themselves to the edge of bistability of a discontinuous phase transition, rather than to the critical point of a continuous one. The equations defining the mathematical theory of SOB turn out to bear a strong resemblance to a (Landau-Ginzburg) theory recently proposed to analyze the dynamics of the cerebral cortex. This theory describes the neuronal activity of coupled mesoscopic patches of cortex, homeostatically regulated by short-term synaptic plasticity. The theory for cortex dynamics entails, however, some significant differences with respect to SOB, including the lack of a (bulk) conservation law, the absence of a perfect separation of timescales and, the fact that in the former, but not in the second, there is a parameter that controls the overall system state (in blatant contrast with the very idea of self-organization). Here, we scrutinize-by employing a combination of analytical and computational tools-the analogies and differences between both theories and explore whether in some limit SOB can play an important role to explain the emergence of scale-invariant neuronal avalanches observed empirically in the cortex. We conclude that, actually, in the limit of infinitely slow synaptic dynamics, the two theories become identical but the timescales required for the self-organization mechanism to be effective do not seem to be biologically plausible. We discuss the key differences between self-organization mechanisms with/without conservation and with/without infinitely separated timescales. In particular, we introduce the concept of "self-organized collective oscillations" and scrutinize the implications of our findings in neuroscience, shedding new light into the problems of scale invariance and oscillations in cortical dynamics.
\end{abstract}

DOI: 10.1103/PhysRevResearch.2.013318

\section{INTRODUCTION}

The theory of self-organized criticality (SOC) explains how systems can become self-organized to the edge of a continuous phase transition, i.e., to the vicinity of a critical point without the apparent need of parameter fine tuning [1-5]. This theory (or mechanism) is often invoked to explain the emergence of scale-free distributions in the sizes and durations of outbursts of activity — often called "avalanches"interspersed by periods of quiescence in diverse settings such as earthquakes, vortices in superconductors [1,3,5], and cortical brain activity [6-9], to name but a few.

The basic mechanism for self-organization to the edge of a phase transition-as exemplified by its most paradigmatic representatives, sandpile models [2,10,11] —relies on two essential and intertwined features: The first one is the presence of two infinitely separated timescales: a fast dynamics characterizes the system intrinsic activity, while a slow

Published by the American Physical Society under the terms of the Creative Commons Attribution 4.0 International license. Further distribution of this work must maintain attribution to the author(s) and the published article's title, journal citation, and DOI. dynamics modulates the control parameter. This latter acts differentially on each phase - at each side of the transitionthus creating a feedback loop that self-organizes the system to the edge of the transition [5,12-14]. The second important feature is that the bulk dynamics is conserved, i.e., dissipation occurs only at the system boundaries: in the presence of bulk dissipation there would be characteristic timescales-both in space and in time-incompatible with the idea of scale invariance.

Nevertheless, self-organized models lacking conservation - such as forest-fire and earthquake models [15,16]- have also a long tradition in studies of self-organized criticality. The main difference between conserved and nonconserved dynamics in SOC models is that while the first one drives the dynamics exactly to the critical point with concomitant scale invariance, nonconserved dynamics leads to a wandering around the critical point (i.e., with excursions to both sides of the critical point but not sitting exactly on it). This mechanism has been termed "self-organized quasicriticality" (SOqC) and leads to approximate scale invariance for a few decades, which may suffice to describe what empirically observed in real systems, without the need to invoke perfect criticality as in SOC (we refer the reader to Bonachela and Muñoz [14] for a thorough and pedagogical discussion of these issues). 
The paradigm of self-organized criticality has been very influential in the context of neuroscience. It has been profusely employed to rationalize the empirical observation of scale-free avalanches, i.e., outbursts of neuronal activity whose sizes and durations are distributed as power laws [17], as robustly found across species, scales, and experimental techniques $[18,19]$. Mathematical models inspired in SOC have been proposed to account for such scale-invariant avalanches $[7,8,20,21]$. Actually, the idea that the cerebral cortex - as well as other biological systems-might extract important functional benefits from operating at criticality, has attracted a lot of interest and excitement as well as some controversy (see, e.g., Refs. [9,18,19,22,23]); SOC, as well as $\mathrm{SOqC}$, played a key role in the development of this conjecture $[21,24]$.

Recently, a mechanism similar in spirit to SOC-relying also on an infinite separation of timescales and on a conserved dynamic for the control parameter-has been discovered to be able to self-organize systems exhibiting a discontinuous phase transition to the edge of such a transition. As a matter of fact, self-organized bistability (SOB) - as the theory has been named-has been proposed as a new and very general paradigm for the self-organization to points of bistability or phase coexistence between two alternative phases [25].

Systems under SOB conditions turn out to be also characterized by scale-free avalanches of activity. More specifically, localized small perturbations into the quiescent state propagate in the form of avalanches with sizes $S$ and durations $T$ distributed as power laws: $P(S) \sim S^{-\tau}$ and $P(T) \sim T^{-\alpha}$, and the averaged avalanche size scales as $\langle S\rangle \sim T^{\gamma}$, obeying the scaling relation $\gamma=(\alpha-1) /(\tau-1)[26,27]$. However, at odds with SOC, avalanches in SOB systems are distributed in a bimodal fashion, i.e., for any finite system size their sizes and times distributions consist of a power-law complemented with a "bump," corresponding to anomalously large system-spanning events [24,25]. Furthermore, the exponents $\tau, \alpha$, and $\gamma$ differ from their SOC counterparts and coincide with those of the usual mean-field branching process exponents even in low dimensional systems [25,28-30].

Given that living systems could have evolved to exploit the complementary benefits of two alternative coexisting phases [9], this new paradigm for the self-organization to the edge of a phase transition has been argued to be of potential relevance in biological problems, much as SOC is. In particular, in the original paper in which SOB was introduced, it was also speculated that SOB might be relevant to explain the emergence of scale-free avalanches in the neuronal activity of the cerebral cortex [25].

During deep sleep or under anesthesia, the state of cortical activity is well-known to exhibit a form of bistability, with an alternation between two possible states of high and low levels of neural activity-called "up and down" states-respectively [31-34]. This underlying bistability, together with the empirical observation of scale-free avalanches-appearing sometimes in concomitance with anomalously large outbursts-in the awake resting brain, made the authors of Ref. [25] suggest (as a possibility to explore) that there could be some type of self-organization to the edge of bistability, rather than the usually postulated self- organization to criticality $[7,8,20,21]$ or any other alternative scenario.

In a parallel endeavor, our research group has recently proposed a physiologically motivated mesoscopic (Landau-Ginzburg) theory, specifically designed to shed light on the large-scale dynamical features of cortical activity [35]. The outcome of such an approach is that the relevant phase transition for cortical dynamics is a synchronization phase transition (which occurs in concomitance with scaleinvariant avalanches) with no self-organization to such a transition: parameters need to be fine tuned to observe it. Moreover, the resulting synchronous-asynchronous phase transition is of a different nature of the quiescent-active phase transition assumed to describe avalanches in cortical dynamics, which has motivated a change of perspective in the field with important consequences.

The most remarkable fact for our purposes here is that the Landau-Ginzburg theory for cortical dynamics bears profound similarities with that of SOB. Thus our main goal here is to scrutinize the analogies and differences between the above Landau-Ginzburg theory and the theory of SOB. In particular, we pose the following question: can self-organization to the very edge of a discontinuous transition with scale-free avalanches be possibly observed in the (Landau-Ginzburg) model? Can the SOB theory be modified to reproduce the phenomenology of the Landau-Ginzburg equation? Answering these questions will pave the way to a deeper understanding of self-organization mechanisms and their relevance in neuroscience.

\section{THE THEORY OF SELF-ORGANIZED BISTABILITY}

Let us overview here the main aspects of the theory of SOB (for the sake of clarity and self-containment, we present in Appendix A a simple one-site or mean-field formulation that might be helpful to gain insight for readers not familiar with SOB or SOC theories; for a complete description of SOB and its mathematical formulation, we refer to di Santo et al. [25]).

Given a spatially extended system, the theory of SOB can be written in terms of two equations, one for an activity field $\rho(\vec{x}, t)$ (which in sandpiles represents sites over the instability threshold) and one for a "background" or "energy" field (which in sandpiles represents the total amount of grains at a given site). ${ }^{1}$

More precisely, the set of Langevin equations proposed to describe the evolution of the activity $\rho(\vec{x}, t)$ and "energy" field $E(\vec{x}, t)$ in the SOB theory [25] read

$$
\begin{aligned}
& \dot{\rho}(\vec{x}, t)=[E(\vec{x}, t)-a] \rho+b \rho^{2}-\rho^{3}+D \nabla^{2} \rho+h+\eta(\vec{x}, t), \\
& \dot{E}(\vec{x}, t)=\nabla^{2} \rho(\vec{x}, t)-\varepsilon \rho(\vec{x}, t)+h
\end{aligned}
$$

[note that some dependencies on $(\vec{x}, t)$ have been omitted in Eq. (1) for simplicity], $a, b>0$ are constants, $\nabla^{2} \rho$ stands for a diffusive coupling with diffusion constant $D$, and $\eta(\vec{x}, t)$ is a zero-mean multiplicative Gaussian noise with $\left\langle\eta(\vec{x}, t) \eta\left(\vec{x}^{\prime}, t^{\prime}\right)\right\rangle=\rho(\vec{x}, t) \delta\left(\vec{x}-\vec{x}^{\prime}\right) \delta\left(t-t^{\prime}\right)$ describing particle-number ("demographic") fluctuations.

\footnotetext{
${ }^{1}$ Note that either name is an abstraction, e.g., in sand piles this field represents the local amount of sand grains.
} 
This is the simplest set of equations extending the theory of self-organized criticality $[5,14,36,37]$ to the case in which there is a discontinuous phase transition [38]. More in particular, the rationale behind these equations is as follows. The first one for the field activity exhibits a discontinuous phase transition for the overall level of activity as the control parameter (e.g., value of $E$ ) is changed. The second one is responsible for creating a feedback loop between the control and the order parameters [12]. When there is no activity, the local energy field grows with the constant driving $+h$ (shifting the system towards the active phase) while, in the presence of activity, dissipation at the boundaries dominates (shifting the system towards the absorbing phase). Importantly, $h$ is an arbitrarily small driving rate, which, in analogy with the driving in sandpile models-where just one sandgrain is added at the time [1] - is assumed to scale with the inverse of the system size $h \propto 1 / L^{2}$. Equivalently $\varepsilon$ is the rate of the activity-dependent energy dissipation, which in sandpile models is a boundary effect, scaling as $\varepsilon \propto 1 / L$; thus, the ratio $h / \varepsilon \rightarrow 0$ in the large system-size limit. In other words, the SOB limit is obtained when driving and dissipation go to zero, i.e., the energy equation converges to be conserved and the timescales of both equations become infinitely separated (in the jargon of nonlinear systems, this corresponds to a "slow-fast" dynamics [39]).

As mentioned in the introduction, the outcome of this set of equations is that the system self-organizes to the point of bistability between the quiescent and the active phase with scale-free avalanches [25]. In particular, the associated exponents $\tau, \alpha$ and $\gamma$ take well-known values, i.e., those of an unbiased branching process [28,40,41] (actually, to be more precise, they coincide with those of compact directed percolation or voter model universality class in all dimensions $[27,42,43]$, and with those of the branching process above the upper critical dimension $d=2$ [25]). Also, as said above, such scale-free distributed avalanches coexist (for any finite system size) with anomalously large events or waves, in which activity spreads ballistically, weeping the whole system [25].

\section{THE LANDAU-GINZBURG THEORY OF CORTEX DYNAMICS}

The set of Eqs. (1) can be directly compared with the physiologically inspired Landau-Ginzburg model that has been recently proposed to shed light on the large-scale features of brain activity, relying on synaptic plasticity as a chief regulatory mechanism [35]. In this modeling approach, neural activity is described at a coarse-grained level in the spirit of the approach of Wilson and Cowan to large-scale neural dynamics $[44,45]$. On the other hand, short-term synaptic plasticity is implemented as a main regulatory mechanismusing the celebrated Tsodyks-Markram model [31] (see also the very similar approach by Abbott et al. [46]) -in line with previously proposed models of self-organization in brain dynamics $[7,8,47,48]$. In particular, the level of neural activity at each coarse-grained or mesoscopic region of the cortical tissue (representing a local subpopulation of neurons) is encoded in an activity variable $\rho(\vec{x}, t)$, while the local amount of available synaptic resources is called $R(\vec{x}, t)$. The following set of equations defines the dynamics of these two variables [35]:

$$
\begin{aligned}
& \dot{\rho}(\vec{x}, t)=(R(\vec{x}, t)-a) \rho+b \rho^{2}-\rho^{3}+I+D \nabla^{2} \rho+\eta(\vec{x}, t) \\
& \dot{R}(\vec{x}, t)=\frac{1}{\tau_{R}}(\xi-R)-\frac{1}{\tau_{D}} R \rho
\end{aligned}
$$

[where, again, some dependencies on $(\vec{x}, t)$ have been omitted for the sake of simplicity]. In the equation for the activity field-much as above- $a, b>0$ are constant parameters, $I$ stands for a small external incoming input, $\nabla^{2} \rho$ stands for the diffusive coupling between local regions with diffusion constant $D$, and $\eta(\vec{x}, t)$ is a multiplicative demographic noise. The bilinear coupling between $R$ and $\rho$ in the first equation reflects the fact that the larger the amount of synaptic resources, the larger the rate at which further activity is generated. On the other hand, in the second equation, $\tau_{R}$ and $\tau_{D}$ are the characteristic scales for the processes of recovery and depletion of synaptic resources, respectively, $\xi$ is the maximal level of available synaptic resources that can possibly be reached.

Let us emphasize that-importantly for what follows - this theory is not self-organized, i.e., the resulting phenomenology depends on the value of the baseline level of synaptic resources $\xi$ :

(1) For small values of $\xi$ the system sets into the quiescent (or "down") state, with vanishing activity.

(2) For large values of $\xi$ the system sets into an active (or "up") state with a sustained (high) level of activity, which is sometimes called "asynchronous irregular" phase.

(3) In between the above two regimes there is an intermediate one called "synchronous irregular" in which there are intermittent events which are synchronized; these correspond to fast waves traveling through the system, and generating coactivation of many units within a relatively small time window.

(4) Right at the critical point separating the synchronous from the asynchronous phase avalanches can be measured by using a protocol-relying on the definition of a discrete time binning-identical to that used by experimentalists to detect neuronal avalanches $[9,17]$.

At the light of these facts, it was proposed that the actual scale-free avalanches of neuronal activity observed in the cortex stem from the dynamics operating in a regime close to the synchronous-asynchronous phase transition, rather than to the critical point of a standard quiescent-to-active phase transition as had been assumed before [18]. However, the mechanism by which the cortex seemingly selects to operate near such a critical point remains undetermined [35].

\section{ANALOGIES AND DIFFERENCES BETWEEN BOTH THEORIES}

Remarkably, the sets of equations (1) and (2) exhibit profound analogies (note that the notation in both of them has been fixed to make the similitudes self-evident). As a matter of fact, the first equation, which describes in the simplest possible (minimal) way a discontinuous/first-order phase transition into an absorbing state, is identical for both theories. On the other hand, formal differences appear in the second equation for the "energy" field in SOB theory, Eq. (1), 
which in Eq. (2) is replaced by a field describing the dynamics of synaptic resources.

A first important difference between both sets of equations is that in SOB the energy field is conserved in the bulk; only driving events and boundary dissipation make the total integral value fluctuate in time, but as specified earlier, they are both vanishingly small. On the other hand, the above equation for $\dot{R}(\vec{x}, t)$ is not conserved in the bulk, as it includes a (positive) term for the charging/recovery of resources to their baseline level $\xi$, as well as a (negative) term for the activity-dependent consumption of resources.

The second (related) difference is that in Eq. (2) there is no perfect separation of timescales. Actually, the synaptic time constants $\tau_{R}$ and $\tau_{D}$ are biologically motivated parameters, e.g., $\tau_{R}=10^{3}$ and $\tau_{D}=10^{2}$ time units [35], that cannot be assumed to scale with the system size (as would be required in SOB) [21].

In third place, in Eq. (2), there is a baseline level of synaptic resources $\xi$ which, as said above, plays the role of an overall control parameter: by tuning it one can shift the system between different phases from a quiescent regime with no activity, to one with oscillations, waves, etc. (see Ref. [35]); such a tuning parameter has no counterpart in SOB, Eq. (1). Indeed, the very existence of a control parameter allowing to shift between dynamical regimes is at odds with the very idea of self-organization.

Thus our aim in what follows is to explicitly explore whether Eq. (2) can lead to the same behavior of SOB-i.e., to true self-organization-in some limit and, also, the other way around: to see what modifications of SOB lead to the Landau-Ginzburg phenomenology.

\section{RESULTS}

Let us start with a single unit (or mean-field) analysis of the local/individual components of Eq. (2) in the stationary state, for which one needs to detect where the two nullclines of the associated deterministic equations (for the one-site dynamics) intersect (see Appendix B for more details as well as Appendix $\mathrm{C}$ for a related model). The fixed points $\left(\rho^{*}, R^{*}\right)$ of the dynamical system depend on the maximal level of synaptic resources, $\xi$. For small values of $\xi$, the deterministic system settles in a "down" state with $\rho^{*}=0$ and $R^{*}=\xi$. In contrast, an "up" state with sustained activity, $\rho^{*}>0$, with a depleted level of resources, $R^{*}<\xi$, emerges for sufficiently large values of $\xi$.

Separating these two limiting states, there is a range of values of $\xi$ where a stable limit cycle emerges if the timescale separation is large enough (see leftmost inset of Fig. 1(a) and purple-shaded region in Fig. 1). As discussed in detail in Appendix B, the limit cycle is created via a homoclinic bifurcation at $\xi=a$ and destroyed via a Hopf bifurcation at a value of $\xi$ that depends on the timescales. In particular, for a biologically plausible separation of the characteristic timescales $\tau_{R}$ and $\tau_{D}$ (i.e., $\tau_{D} / \tau_{R}=0.1$ and $\tau_{R}=10^{3}$ as chosen in Ref. [35]) the system exhibits an intermediate regime with oscillations as illustrated in Fig. 1(a) and Appendix B. These oscillations at the individual sites are at the origin of the propagating waves and emerging synchronous behavior in the spatially extended system [35]. On the other hand, in the

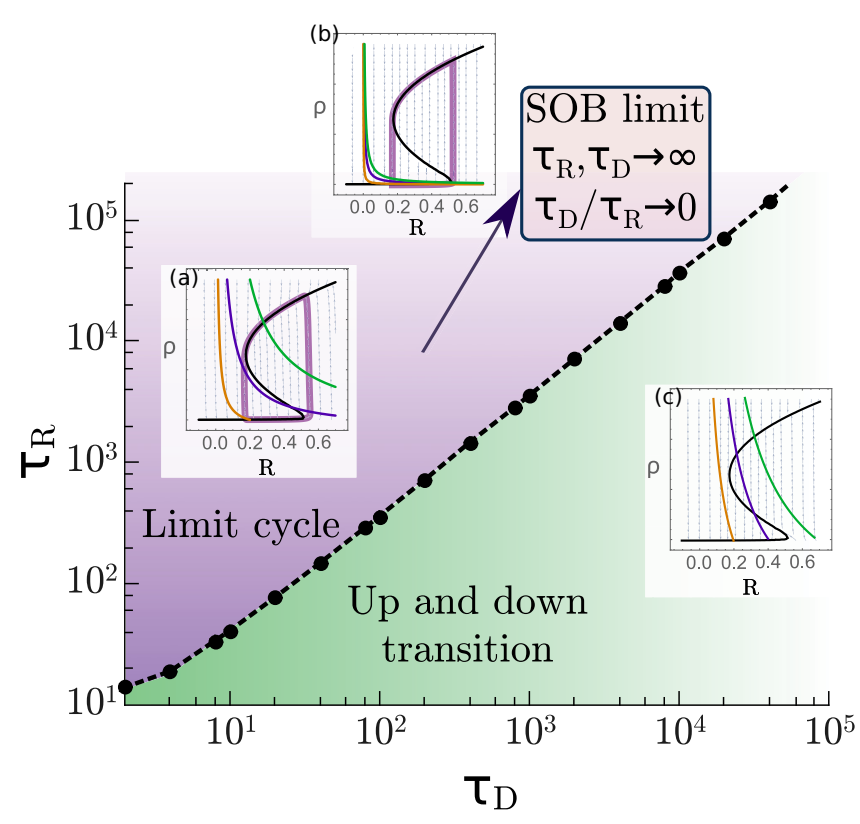

FIG. 1. Phase portraits and nullclines for the deterministic dynamics at an individual site of the Landau-Ginzburg theory, Eq. (2). Insets: the nullcline for $\dot{\rho}=0$ is colored in black, while nullclines for $\dot{R}=0$-for three different values of the baseline level of synaptic resources $\xi$ - are plotted in orange, purple, and green color, respectively. The small grey arrows represent the vector field for $(\dot{\rho}, \dot{R})$ and the light purple curve represents a limit-cycle trajectory. [Inset (a)] For $\tau_{D} / \tau_{R}=0.1$ and $\tau_{R}=10^{3}$, the system may display a down state (orange nullcline, $\xi=0.2$ ), a limit cycle (purple nullcline, $\xi=1.5$ ) or an up state (green nullcline, $\xi=3.5$ ). [Inset (b)] For $\tau_{D} / \tau_{R}=$ 0.001 and $\tau_{R}=10^{7}$ the system approaches the SOB behavior and the dependence on the control parameter $\xi$ becomes very weak, meaning that for a wide range of values of the control parameter above the down state $(\xi=1,5,10$ for orange, purple, and green nullclines, respectively) the system sets in the oscillatory phase. [Inset (c)] When the separation of timescales is very low, the system only displays bistability between up and down states with no oscillations whatsoever, no matter the value of $\xi$. (Main) The global behavior of the system depending on the timescale separation is coded in the main figure with different background colors: purple when the limit cycle appears, and green when only bistability is possible. Parameters are set to $I=10^{-3}, a=0.6, b=1.3$, in both cases.

opposite limit in which the timescales are both relatively fast and not very separated - a case that we do not discuss here in further detail-there is no intermediate regime of oscillations and the system just shifts from a quiescent (down) to an active (up) state as $\xi$ is increased [see the green-shaded region in Fig. 1 and inset (c)]. This regime is likely to be useful to describe up-and-down transitions as observed in the cerebral cortex.

Given that the mechanism of the recovery of synaptic resources (with a characteristic timescale $\tau_{R}$ ) plays the role of driving, while the depletion of resources (with a characteristic scale $\tau_{D}$ ) plays the role of dissipation in presence of neural activity, we conjecture that the SOB limit of infinite separation of timescales could be only possibly recovered taking formally the double limit $1 / \tau_{R} \rightarrow 0$ and $1 / \tau_{D} \rightarrow 0$, with $\tau_{D} / \tau_{R} \rightarrow 0$. In particular, in what follows we consider different sets of values for the timescales $\tau_{R}$ 

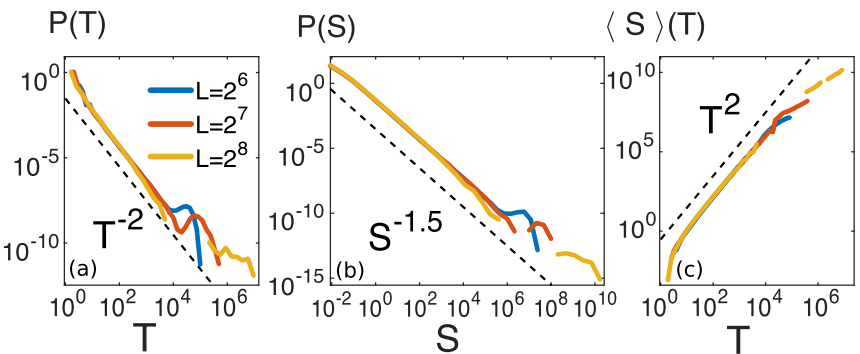

FIG. 2. Avalanches in the limit of a large separation of timescales, for the spatially extended (two dimensional) version of the Landau-Ginzburg theory for cortical dynamics, Eq. (2). Probability distribution for avalanche durations $T$ (a), avalanche sizes $S$ (b) and average avalanche size as a function of duration (c) in double logarithmic scale, for square-lattice systems of sizes: $N=2^{12}, 2^{14}$, and $2^{16}$. The dashed lines are plotted as a guide to the eye, and have slopes corresponding to the expectations for an unbiased branching process $(\alpha=2, \tau=3 / 2$, and $\gamma=2$, respectively). The "bumps" correspond to anomalously large events, i.e., synchronized spiking events. The cut-offs/bumps change with $N$ obeying finite-size scaling as in the theory of self-organized bistability [25]. Parameters: $b=0.5 a=1, I=10^{-7}, \tau_{D}=10^{4}, \tau_{R}=10^{6}$, and $D=1$.

and $\tau_{D}$-regardless of their real meaning in neuroscienceapproaching a large separation of timescales [e.g., $\tau_{D} / \tau_{R}=$ 0.001 rather than $\tau_{D} / \tau_{R}=0.1$ as above; see Fig. 1(b)].

First of all, we observe that the larger the separation of timescales, the weaker the dependence on the control parameter $\xi$ [see in particular in Fig. 1(b) how the three different $\dot{R}=0$ nullclines-for three different values of $\xi$-become very close to each other]. This allows us to reproduce one of the main features of the self-organization mechanism: robustness on changes of the tuning parameter emerges as the limit of infinite separation of timescales is approached (see Appendix B for more details).

Second, when the separation of timescales tends to infinity, the slope of the nullcline $\dot{R}=0$ converges to zero at the intersection point. Then, both nullclines intersect in a tangential way, giving rise to a limit cycle, much as in the mean-field SOB (see Appendix A).

To go beyond the single-site or mean-field analysis we now study computationally a full (spatially explicit) system. Under the conditions of a large separation of timescales we have performed computer simulations of the full set of Eqs. (2). In particular, we considered square lattices with sizes from $N=2^{12}$ to $2^{16}$ to analyze finite size effects and performed up to $10^{8}$ runs for different parameter sets. It is possible to measure avalanches of activity by defining a small threshold (e.g., $\theta=10^{-6}$ ) for the overall (integrated) activity and analyzing the statistics of excursions (sizes $S$ and times $T$ ) above such a threshold [25,41]. Results of our extensive computer simulations are shown Figs. 2 and 3.

In particular, Fig. 2 reveals the existence of scale-invariant episodes of activity - whose distributions are very well fitted by the exponents of the unbiased branching process- appearing together with anomalous "king" avalanches in full analogy with the theory of SOB [25]. These computational results are very robust to changes in the control parameter $\xi$ as illustrated in Fig. 3. Actually, hardly any difference is observed in the probability distributions when $\xi$ is increased from a value 1 to 10 . This reveals the emergence of true self-organization regardless of the specific value of the parameters in the limit in which an infinite separation of timescales is imposed.

Thus, at the steady state, the system with infinitely separated timescales actually self-organizes to the edge of a discontinuous/first-order phase transition, where active and absorbing phases coexist and scale-invariant episodes of activity emerge, as in SOB.

Finally, observe that the lack of a diffusion term in the equation for synaptic resources does not seem to be a problem, indicating that diffusion in the second equation is not essential ("relevant" in the jargon of the renormalization group) feature and that possibly it could also be removed from the minimal set of Eqs. (1) describing SOB (this claim is also supported by our own computational analyses).

\section{DISCUSSION}

We have shown, by using the recently proposed LandauGinzburg model of cortex dynamics [35], that selforganization behavior to the edge of a discontinuous phase transition with bistability can be recovered by considering extremely slow synaptic timescales.

First, from a theoretical point of view, our analyses reveal that different regulatory mechanisms for the control
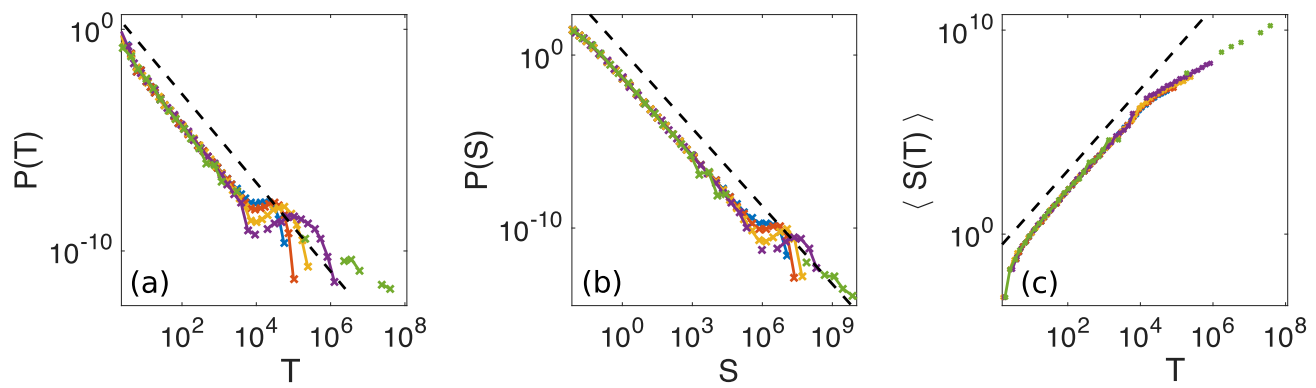

FIG. 3. Weak dependence on the control parameter in the limit of large separation of timescales, for the spatially extended (two dimensional) version of the Landau-Ginzburg theory for cortical dynamics, Eq. (2). Different colors represent different values of $\xi$. There is essentially no visible change in the probability distributions for avalanche durations $T$ (a), avalanche sizes $S$ (b), and average avalanche size as a function of duration (c), for different values of $\xi$, in particular, $\xi=1,3,5,7$, and 10. The dashed lines are plotted as a guide to the eye and their slopes correspond to unbiased branching process exponents. Parameters are fixed as in Fig. 2 with $N=2^{14}$. 
parameter-i.e., different equations for the "energy" field, with different meanings and possibly with diverse features such as conserved dynamics or not, or with or without a diffusion term-can be considered in the context of self-organization to the neighborhood of a discontinuous transition. The SOB phenomenology-which is characterized by scale-free avalanches controlled by branching process exponents, appearing together with anomalously large avalanches-emerges once the "self-organization limit" is taken: in the case considered here this means that the dynamics of charging and discharging synapses needs to be infinitely slow.

Our approach allows us to illustrate that, one can use diverse dynamical models to recover scale-free distributions of neuronal avalanches - with the empirically observed branching-process exponents - but, the synaptic timescales needed to recover them in the limit of SOB are not compatible with those of a realistic short term plasticity dynamics. Indeed, as reported by neurophysiological measurements, realistic synaptic timescales are comprised between few hundreds of milliseconds up to few seconds [31,46,49,50], which are clearly far from the SOB limit, requiring a much slower dynamics. For instance, the unit of time, in the Landau-Ginzburg model, should be understood in terms of the spontaneous decay of neural activity: one time unit in Eq. (2) is of the order of the millisecond, which means that in order to recover the SOB limit, the recovery timescale for synaptic resources should be of the order of several minutes! On the other hand, the Landau-Ginzburg model-leading to verifiable predictionsis based on the consideration of realistic timescales. In other words, the "imperfect" form of SOB, with finite time scales is much more adequate to describe neural dynamics than SOB itself.

Thus self-organization to the edge of bistability can be achieved only by considering an infinite separation of timescales that does not seem to be plausible in the cortex, at least not by considering short-term plasticity as a chief regulatory mechanism. Importantly, the same criticism can be made — and has been explicitly made [21] — to models for neural dynamics based on self-organized criticality: they also require an infinite separation of timescales to work. In order to circumvent, this conceptual problem in SOC, i.e., to deal with real systems that are not perfectly conservative nor operate under the strict condition of perfectly separated timescalesas mentioned above-researchers introduced the concept of self-organized quasi criticality (SOqC). Such quasicritical systems self-organize to operate within a neighborhood of the critical point, with continuous excursions to both sides. In other words, when the strict mathematical conditions for SOC are relaxed, the resulting self-organized systems are not perfectly critical nor show perfect scale invariance. Rather they hover around the critical point and exhibit imperfect scaling [14].

Thus, in full analogy with $\mathrm{SOqC}$, we propose that in order to describe "imperfect" SOB systems, such as the Landau Ginzburg theory discussed here, one should use a terminology, such as "self-organized quasi bistability," or better, "self-organized collective oscillations": if the separation of timescales is not large, then-depending on the control parameter value - the system is either in a down state, up state, or in an intermediate range of large waves or collective oscillations. However, as the separation of timescales is progressively increased, the system becomes more and more SOB-like, with scale-free avalanches and progressively less frequent large waves of activity (see Eq. (2) as well as Refs. [51,52]).

From the viewpoint of neuroscience, it is important to recall that the original Tsodyks-Markram model for short-time synaptic plasticity implements an additional variable describing a "facilitation" mechanism whose timescale could be much longer than the depression one considered here $[31,46,53]$. This would make the dynamics more complex and eventually regulate the baseline of synaptic resources, allowing self-tuning to the transition point. Also, one could explore the role played by other types of plasticities, including facilitation, such as spike-timing dependent plasticity. In particular, plasticity operates in the cortex through a wide spectrum of timescales. For example, long term potentiation is known to involve different mechanisms, such as the production of new ion channels, modifying the excitability of the units for a long-lasting period. It is likely that for homeostatic mechanisms with larger separation of timescales, behavior closer to SOB could emerge.

Actually, in order to illustrate the generality of the discussions, here we have conducted similar analyses on a similar model for neural dynamics in the presence of adaptation (rather than synaptic plasticity), recently introduced by Levenstein et al. (see Appendix C). This research line seems promising and we leave a careful exploration of this possibility for a future work.

Summing up, self-organized bistability with its concomitant scale-free avalanches of activity can be obtained as a limiting case of the Landau-Ginzburg model for cortex dynamics. However, this only occurs in the unrealistic limit of an extremely slow dynamics for synaptic resources is considered. On the other hand, relaxing the strict conditions for SOB (i.e., not imposing a huge separation of timescales) one obtains a version of the SOB theory - that have also very remarkable features - and that we call self-organized quasi bistability, or better, "self-organized collective oscillations," that can be much more adequate to describe real systems, e.g., in neuroscience.

\section{ACKNOWLEDGMENTS}

We acknowledge the Spanish Ministry and Agencia Estatal de investigación (AEI) through grant FIS2017-84256P (European Regional Development Fund), as well as the Consejería de Conocimiento, Investigación y Universidad, Junta de Andalucía and European Regional Development Fund (ERDF), Ref. SOMM17/6105/UGR for financial support. We also thank Cariparma for their support through the TEACH IN PARMA project.

\section{APPENDIX A: MEAN-FIELD APPROACH TO SOB}

Here we perform a detailed single-site or mean-field analysis of the dynamical system describing SOB, i.e., Eqs. (1) but neglecting spatial dependence and noise. The first equation in 


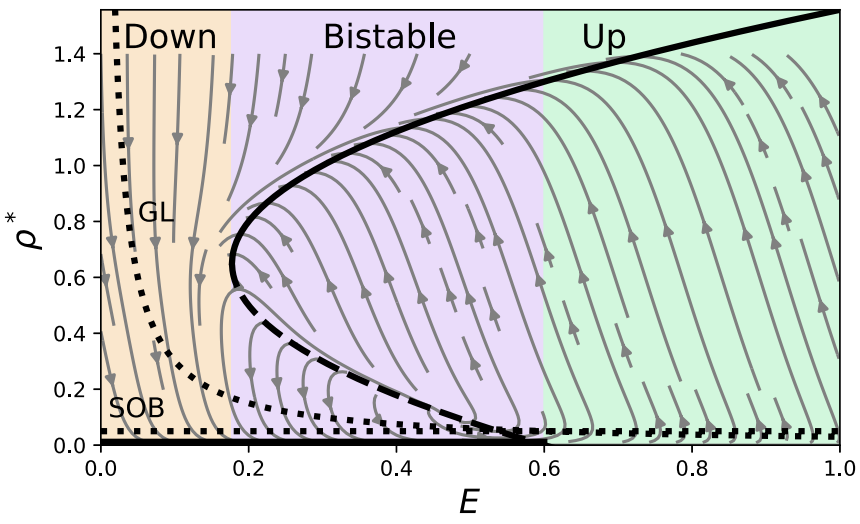

FIG. 4. Two forms of self-organization. Diagram showing the steady-state solutions $\rho^{*}$ of Eq. (A2) as a function of $E$. The solid line indicates stable fixed points, while the dashed line indicates unstable ones. Taking $E$ as a control parameter there are three different regions: a down state, a bistability region, and an up state. When a dynamics for $E$ is added, the system may display oscillations. For the sake of comparison, nullclines are displayed for both the SOB, Eq. (A3) (horizontal dotted line) and the Landau-Ginzburg for the cortex, Eq. (B1) (curved dotted line) models. Note that the nullclines in these two cases tend to cut tangentially at the bifurcation point, $\xi=a$-becoming locally indistinguishable-as the large timescale separation limit is approached. Gray arrows represent the corresponding vector field for the case of the LandauGinzburg model. Parameters have been set to $a=0.6, b=1.3, h=$ $0.005, \varepsilon=0.1$, and $\xi=1.0$.

Eqs. (1) can then be simply written as

$$
\dot{\rho}=(-a+E) \rho+b \rho^{2}-\rho^{3}+h,
$$

where now $\rho$ is the overall density of activity. Let us analyze this equation for $\dot{E}=0$, i.e., for a fixed value of $E$, with vanishing driving $h$ and dissipation $\varepsilon$. Then Eq. (A1) has the typical form of a discontinuous transition for $\rho$. Actually, imposing stationarity, $\dot{\rho}=0$, one obtains three possible solutions: $\rho_{0}=0$ (stable for $E<a$ ) and a positive and negative pair,

$$
\rho_{ \pm}=\frac{b}{2} \pm \sqrt{\frac{b^{2}}{4}+(-a+E)}
$$

emerging at a saddle-node bifurcation. As illustrated in Fig. 4, these last ones exist (as real solutions) only for $E>a-b^{2} / 4$ and $\rho_{+}$is stable for $a-b^{2} / 4<E<a$. On the other hand, $\rho_{-}$is unstable in this region, and becomes nonphysical, i.e., negative for $E>a$. This gives a bistability region (shaded in magenta color in Fig. 4) where two stable fixed points $\rho_{0}$ and $\rho_{+}$coexist, with a branch of unstable fixed points $\rho_{-}$in between.

These are the solutions of the problem for constant "energy" $E$. For the full system, including the dynamics for $E$ (i.e., for $h \neq 0, \varepsilon \neq 0$ ) the mean-field dynamics of the control parameter $E$ is given by

$$
\dot{E}=h-\varepsilon \rho .
$$

Observe that this dynamics acts differentially depending on the phase, i.e., on the value of $\rho$ : in the absence of activity it increases owing to the driving $h$, while as activity grows the dissipation (negative) dominates the dynamics. The only possible fixed point - where these two contributions balanceis $\rho^{*}=h / \varepsilon$. Observe that, this equation defines a nullcline which is a horizontal line in Fig. 4. Note also that in the double limit of conservation $h, \varepsilon \rightarrow 0$ with an infinite separation of timescales $h / \varepsilon \rightarrow 0$, this is necessarily a point of vanishingly small activity and, as seen above (see Fig. 4), the branch of points with low activity is unstable. Indeed, from Eq. (A2) at stationarity, one obtains $E^{*}=a-b h / \varepsilon+(h / \varepsilon)^{2}$, which corresponds to an unstable spiral focus. Inspection of the associated velocity field reveals that the dynamics exhibits a stable limit cycle around such an unstable fixed point (see Fig. 4), shifting cyclically between the upper and the lower branches, with high and low activity, respectively. Note that in the limit $h / \varepsilon \rightarrow 0$, the timescale between succesive cycles increases (in particular, the imaginary part of the eigenvalues associated with the unstable spiral $\left(\rho^{*}, E^{*}\right)$, scales as $\sim(h / \varepsilon)^{-1}$, meaning that the frequency of oscillations goes to 0 as happens in an infinite period bifurcation [54]).

This simple mean-field approach shows that the system behaves as an excitable system in which following external driving there are cycles of periodic activity. As shown in detail in Ref. [25], going beyond mean-field-i.e., turning back to a spatially explicit system such as a lattice-this mechanism generates scale-free avalanches alternating with system-wide spanning waves of activity. The reason behind such a change is that in the above situation, driving may induce local jumps of the activity from the lower branch to the upper one, and from this it can propagate to nearest neighbors coupled to it, giving rise to a local avalanche of activity [25].

\section{APPENDIX B: MEAN-FIELD APPROACH TO THE LANDAU-GINZBURG THEORY}

Here, we discuss the single site or mean-field description of the Landau-Ginzburg theory for cortex dynamics. The first single-unit equation is identical to Eq. (A1), while the second one reads

$$
\dot{E}=\frac{1}{\tau_{R}}(\xi-E)-\frac{1}{\tau_{D}} E \rho .
$$

where $E$ is now the overall "energy" or density of synaptic resources. We call it here $E$ rather than $R$ as in Eq. (2) to make a more direct comparison with Appendix A; also to make the parallelism with the SOB theory even more explicit, we define $h=1 / \tau_{R}$ and $\varepsilon=1 / \tau_{D}$, and study the double limit of slow synaptic dynamics $h, \varepsilon \rightarrow 0$ with $\Delta \equiv h / \varepsilon \rightarrow 0$, in which the two synaptic timescales are infinitely separated.

The main difference between this mean-field theory and its SOB counterpart (in Appendix A) is the existence in Eq. (B1) of a key parameter $\xi$, which bounds the largest possible value of $E$. Three cases can be distinguished depending on the value of $\xi$ and the separation of timescales.

(i) If $\xi<a$ obviously $E<a$, and then equation (A2) has a negative linear term, implying that $\rho=0$ is the only possible stable state. In other words, the two nullclines intersect in the absorbing phase, i.e., for $\rho=0$ [see orange line in Fig. 1(a)].

(ii) More in general, imposing $\dot{E}=0$, one finds the fixed point value $E^{*}=\frac{h \xi}{(h+\varepsilon) \rho}($ defined only for $\rho>0)$ which in the limit of large timescale separation becomes $E^{*} \simeq \frac{\Delta \xi}{\rho}$, 


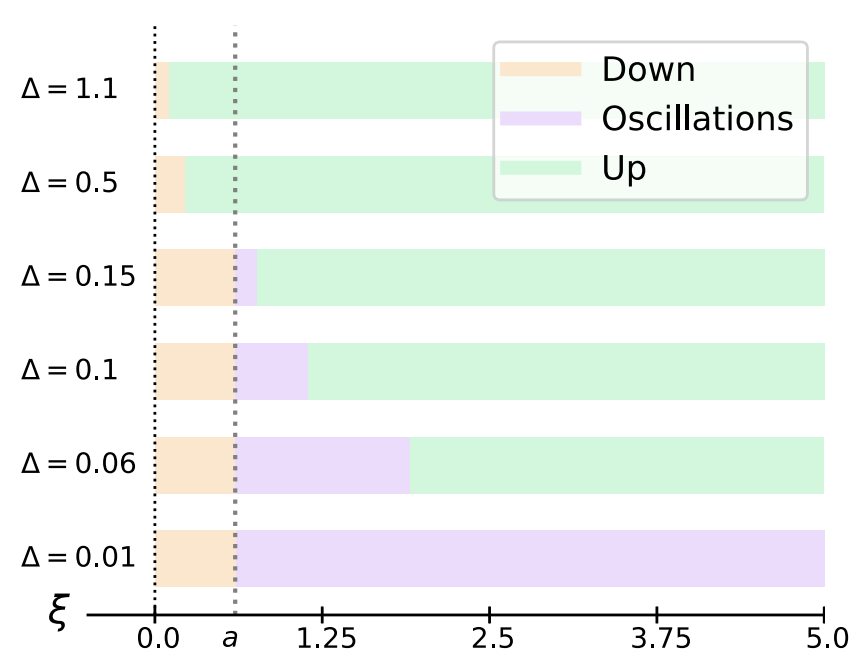

FIG. 5. Invariance on $\xi$. Behaviour of the Landau-Ginzburg system as a function of the control parameter $\xi$, as the limit $\Delta \rightarrow 0$ is approached. Each color represents a different phase (see legend). Note that as the separation of timescales increases (i.e., as $\Delta \rightarrow 0$, in the SOB limit) the oscillatory phase takes more space until it comprises the whole region $\xi>a$. Parameter values: $a=0.6, b=1.3$.

implying that the nullclines intersect at an unstable fixed point (see red line in the leftmost inset of Fig. 1), after a homoclinic bifurcation at $E=a$. This leads to oscillations in the following way: within the absorbing state, energy slowly increases as $\dot{E}=h(\xi-E)$ and-if $\xi>a$ - at some point the system reaches the value $E=a$ where the absorbing state $\rho=$ 0 becomes unstable and activity grows. Then, the energy fixed point is given by $E^{*}$. If the energy is larger than this value, it decreases in a fast way, until $E=E^{*}$. However, if $\Delta$ is very small, the fixed point is $E^{*} \simeq 0$, meaning that $E$ decreases until the up-state branch becomes unstable again-falling to the absorbing state. When the absorbing state is reached, $E^{*}$ is unstable and the cycle starts again. This simple back-and-forth mechanism is responsible for the emergence of limit cycles in the dynamics for $\xi>a$ (see also Ref. [32] where a mechanism very similar to this is termed "Sisyphus effect"). This type of effect is well-known in the theoretical-neuroscience literature (see e.g., Refs. [33,34,49,55-60]).

(iii) For larger values of $\xi$, such that the nullclines intersect in the (stable) up-state branch (see the brown line in the leftmost inset of Fig. 1) there are no oscillations and the system sets in an active state with $E^{*}=\frac{\Delta \xi}{\rho}$ (the oscillations disappear at a Hopf bifurcation). Observe that the condition for this case (iii) to emerge depends on the separation of timescales, $\Delta$. In particular, in the limit $\Delta \rightarrow 0$, a value $\xi \rightarrow+\infty$ is required to destabilize the cycle limit. This effect is illustrated in Fig. 5, where it can be seen that as the $\Delta \rightarrow 0$ limit is approached the regime of oscillations broadens (up to infinite).

Finally, let us note that in case (ii) - which is the one for which avalanches are obtained once the spatially explicit version of the model is considered-the nullcline associated with Eq. (B1) becomes very similar to its counterpart for the SOB case: both of them intersect the unstable branch of $\rho$ solutions in the same part of it and with flat slopes in both cases in the limit $\Delta \rightarrow 0$. Also, observe in the upper inset of
Fig. 1 that the nullclines are quite insensitive to the specific value of $\xi$ (as long as $\xi>a$ ) when such a limit is taken. Thus the same phenomenology is expected to emerge in both cases.

\section{APPENDIX C: NEURAL MODEL WITH ADAPTATION}

The ideas exposed above can be applied to models of neural activity relying on regulatory mechanisms other than synaptic plasticity. To illustrate this, here we consider a model recently proposed by Levenstein et al. [60] to describe cortical dynamics. The model is defined by two differential equations, one for the spike rate $r$ of a cortical mesoscopic region and another one for an "adaptation variable" $a$ that regulates the spiking rate; in particular:

$$
\begin{gathered}
\dot{r}=-r+S\left(\omega r-b a+I, 1, I_{0}\right), \\
\dot{a}=-a+S\left(r, k, r_{0}\right),
\end{gathered}
$$

where $S\left(x, k, x_{0}\right)=1 /\left[1+\exp \left(-k\left(x-x_{0}\right)\right)\right]$ is a sigmoidal function whose steepness is controlled by the parameter $k$, $x_{0}$ is a threshold, $I$ the external input, and $\omega$ and $b$ are the coupling and the adaptation strengths, respectively.

The equation for the mean rate is very similar to the corresponding one for the Landau-Ginzburg theory Eq. (A1); actually, truncating the series expansion of $S$ around the threshold $I_{0}$, transforms the first equation into a third-degree polynomial, exhibiting also a discontinuous transition as Eq. (A1).

To ease the comparison between Eq. (C2) and the LandauGinzburg theory given by Eq. (2), we perform a change of variables $E=1 / a$. Expanding in power series in $r-r_{0}$ and truncating up to leading order, the adaptation equation becomes $\dot{E}=E\left[1-E\left(2+k\left(r-r_{0}\right)\right) / 4\right]$. Observe that here $E=1 / a$ plays a role analogous to that of the synaptic resources in the Landau-Ginzburg theory, and that the similitude between both theories (at a mean-field level) is self-evident.

Notice also that the energy charge (positive term) is of order unity, while the discharge (negative) grows with $k$; thus, taking the limit $k \gg 1$ of very steep adaptation and taking the whole adaptive dynamics to be arbitrarily slow, leads to

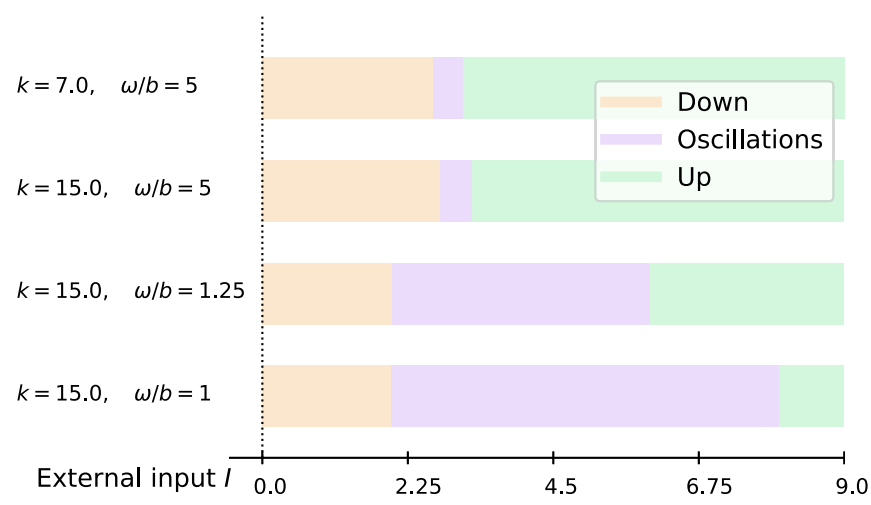

FIG. 6. Width of the region in which collective oscillations emerge in the model for adaptation model of Levenstein et al. [60]. It shows that either increasing $k$ or, alternatively, decreasing the ratio $\omega / b$ enhances the region of collective oscillation, as in Fig. 5. Taking, either of these combinations of parameters to their limit $k \rightarrow \infty$ or $\omega / b \rightarrow 0$ one observes a huge range for self-organized collective oscillations. 
similar effect as the infinite separation of timescales limit in the case of the Landau-Ginzburg theory.

An alternative, more efficient, way to achieve the limit of self-organization is to modify the coupling and the adaptation strength variables $(\omega$ and $b)$. Actually, our Fig. 1 is very similar to Fig. 3 B in Ref. [60], where the role of the timescales $\tau_{R}$ and $\tau_{D}$ is played by $\omega$ and $b$. This implies that taking the limit $\omega / b \rightarrow 0$ while increasing both variables should enlarge the oscillatory region in the same way that happens with the nonconserved SOB and Landau-Ginzburg theory (see Appendices A and B). However, the SOB limit of perfect self-organization cannot be reached in this model, as there is no absorbing or quiescent state.
We performed numerical simulations of Eq. (C2) and measured the length of the oscillatory region as a function of parameters ( $k$ on the one hand or $\omega$, and $b$ on the other). We find-as illustrated Fig. 6-that the width of such a region increases as the separation of timescales is enlarged (much as in Fig. 5).

Thus self-organized collective oscillations emerge in a rather generic way for reasonable parameter choices in this model. This result illustrates that the ideas discussed before for neural dynamics and self-organization are not specific of the Landau-Ginzburg theory relying on short-term synaptic plasticity, but can be also extended to other models of neural dynamics.
[1] P. Bak, How Nature Works: The Science of Self-Organized Criticality (Copernicus, New York, 1996).

[2] P. Bak, C. Tang, and K. Wiesenfeld, Phys. Rev. Lett. 59, 381 (1987).

[3] G. Pruessner, Self-Organised Criticality (Cambridge University Press, Cambridge, 2012).

[4] H. J. Jensen, Self-Organized Criticality: Emergent Complex Behavior in Physical and Biological Systems (Cambridge University Press, Cambridge, 1998).

[5] R. Dickman, M. A. Muñoz, A. Vespignani, and S. Zapperi, Braz. J. Phys. 30, 27 (2000).

[6] D. R. Chialvo, Phys. A 340, 756 (2004).

[7] A. Levina, J. M. Herrmann, and T. Geisel, Nat. Phys. 3, 857 (2007).

[8] D. Millman, S. Mihalas, A. Kirkwood, and E. Niebur, Nat. Phys. 6, 801 (2010).

[9] M. A. Muñoz, Rev. Mod. Phys. 90, 031001 (2018).

[10] S. S. Manna, J. Phys. A: Math. Gen. 24, L363 (1991).

[11] K. Christensen, A. Corral, V. Frette, J. Feder, and T. Jøssang, Phys. Rev. Lett. 77, 107 (1996).

[12] L. Moreau and E. Sontag, Phys. Rev. E 68, 020901(R) (2003).

[13] G. Grinstein, in Scale Invariance, Interfaces, and NonEquilibrium Dynamics, edited by A. McKane et al., NATO Advanced Study Institute, Series B: Physics Vol. 344 (Plenum Press, New York, 1995).

[14] J. A. Bonachela and M. A. Muñoz, J. Stat. Mech. (2009) P09009.

[15] B. Drossel and F. Schwabl, Phys. Rev. Lett. 69, 1629 (1992).

[16] Z. Olami, H. J. S. Feder, and K. Christensen, Phys. Rev. Lett. 68, 1244 (1992).

[17] J. M. Beggs and D. Plenz, J. Neurosci. 23, 11167 (2003).

[18] D. Plenz and E. Niebur, Criticality in Neural Systems (John Wiley \& Sons, New York, 2014).

[19] L. Cocchi, L. L. Gollo, A. Zalesky, and M. Breakspear, Prog. Neurobiol. 158, 132 (2017).

[20] L. de Arcangelis, C. Perrone-Capano, and H. J. Herrmann, Phys. Rev. Lett. 96, 028107 (2006).

[21] J. Bonachela, S. de Franciscis, J. Torres, and M. A. Muñoz, J. Stat. Mech. (2010) P02015.

[22] D. R. Chialvo, Nat. Phys. 6, 744 (2010).
[23] O. Kinouchi and M. Copelli, Nat. Phys. 2, 348 (2006).

[24] O. Kinouchi, L. Brochini, A. A. Costa, J. G. F. Campos, and M. Copelli, Sci. Rep. 9, 3874 (2019).

[25] S. di Santo, R. Burioni, A. Vezzani, and M. A. Muñoz, Phys. Rev. Lett. 116, 240601 (2016).

[26] O. Perković, K. Dahmen, and J. P. Sethna, Phys. Rev. Lett. 75, 4528 (1995).

[27] M. A. Muñoz, R. Dickman, A. Vespignani, and S. Zapperi, Phys. Rev. E 59, 6175 (1999).

[28] T. E. Harris, The Theory of Branching Processes (Courier Corporation, Chelmsford, 2002).

[29] T. Liggett, Interacting Particle Systems, Classics in Mathematics (Springer, Berlin, 2004).

[30] H. W. Watson and F. Galton, J. R. Anthropol. Inst. 4, 138 (1875).

[31] D. Holcman and M. Tsodyks, PLoS Comput. Biol. 2, e23 (2006).

[32] K. Mikkelsen, A. Imparato, and A. Torcini, Phys. Rev. Lett. 110, 208101 (2013).

[33] P. E. Latham, B. Richmond, P. Nelson, and S. Nirenberg, J. Neurophysiol. 83, 808 (2000).

[34] M. Giugliano, P. Darbon, M. Arsiero, H.-R. Luscher, and J. Streit, J. Neurophysiol. 92, 977 (2004).

[35] S. di Santo, P. Villegas, R. Burioni, and M. Muñoz, Proc. Natl. Acad. Sci. USA 115, E1356 (2018).

[36] A. Vespignani, R. Dickman, M. A. Muñoz, and S. Zapperi, Phys. Rev. Lett. 81, 5676 (1998).

[37] A. Vespignani, R. Dickman, M. A. Muñoz, and S. Zapperi, Phys. Rev. E 62, 4564 (2000).

[38] P. Villa Martín, J. A. Bonachela, S. A. Levin, and M. A. Muñoz, Proc. Natl. Acad. Sci. USA 112, E1828 (2015).

[39] M. Desroches, B. Krauskopf, and H. M. Osinga, Nonlinearity 23, 739 (2010)

[40] S. Zapperi, K. B. Lauritsen, and H. E. Stanley, Phys. Rev. Lett. 75, 4071 (1995).

[41] S. di Santo, P. Villegas, R. Burioni, and M. A. Muñoz, Phys. Rev. E 95, 032115 (2017).

[42] J. Marro and R. Dickman, Nonequilibrium Phase Transitions in Lattice Models, Collection Aléa-Saclay (Cambridge University Press, Cambridge, 2005).

[43] M. Henkel, H. Hinrichsen, and S. Lübeck, Non-Equilibrium Phase Transitions: Absorbing Phase Transitions, Theoretical 
and Mathematical Physics (Springer, London, Berlin, 2008).

[44] H. R. Wilson and J. D. Cowan, Biophys. J. 12, 1 (1972).

[45] J. D. Cowan, J. Neuman, and W. van Drongelen, J. Math. Neurosci. 6, 1 (2016).

[46] L. F. Abbott, J. Varela, K. Sen, and S. Nelson, Science 275, 221 (1997).

[47] A. Levina, J. M. Herrmann, and T. Geisel, Phys. Rev. Lett. 102, 118110 (2009).

[48] L. de Arcangelis, Eur. Phys. J.: Spec. Top. 205, 243 (2012).

[49] M. Mattia and M. V. Sanchez-Vives, Cogn. Neurodyn. 6, 239 (2012).

[50] G. Buzsaki, Rhythms of the Brain (Oxford University Press, Oxford, 2009).

[51] R. Burioni, S. di Santo, M. di Volo, and A. Vezzani, Phys. Rev. E 90, 042918 (2014).

[52] F. Pittorino, M. Ibáñez-Berganza, M. di Volo, A. Vezzani, and R. Burioni, Phys. Rev. Lett. 118, 098102 (2017).
[53] J. M. Cortes, M. Desroches, S. Rodrigues, R. Veltz, M. A. Muñoz, and T. J. Sejnowski, Proc. Natl. Acad. Sci. USA 110, 16610 (2013).

[54] S. Strogatz, Nonlinear Dynamics and Chaos: With Applications to Physics, Biology, Chemistry, and Engineering, Studies in Nonlinearity (Westview Press, Cambridge, 1994).

[55] M. Bazhenov, I. Timofeev, M. Steriade, and T. J. Sejnowski, J. Neurosci. 22, 8691 (2002).

[56] A. Compte, M. V. Sanchez-Vives, D. A. McCormick, and X.-J. Wang, J. Neurophysiol. 89, 2707 (2003).

[57] G. Gigante, M. Mattia, and P. Del Giudice, Phys. Rev. Lett. 98, 148101 (2007).

[58] C. Curto, S. Sakata, S. Marguet, V. Itskov, and K. D. Harris, J. Neurosci. 29, 10600 (2009).

[59] J. Tabak, J. Rinzel, and R. Bertram, PLoS Comput. Biol. 7, e1001124 (2011).

[60] D. Levenstein, G. Buzsáki, and J. Rinzel, Nat. Commun. 10, 2478 (2019). 